\title{
On a multi-dimensional risk model with regime switching
}

\author{
Guanqing Wang ${ }^{a, *},{\text { Guojing } \text { Wang }^{b}, \text { Hailiang Yang }}^{a}$ \\ ${ }^{a}$ Department of Statistics and Actuarial Science, \\ University of Hong Kong, Pokfulam Road, Hong Kong \\ ${ }^{b}$ Center for Financial Engineering and Department of Mathematics, \\ Soochow University, Suzhou 215006, P.R.China
}

\begin{abstract}
We consider an insurer with $n(n \geq 2)$ classes of insurance business. The surplus process for each class of insurance business is assumed to follow a compound Cox risk process. Assume that $n$ surplus processes are correlated with thinning dependence and regime switching . By summing up the $n$ surplus processes we obtain a correlated risk process. Upper bounds for the ruin probability under certain assumptions are derived. The joint ruin probability for $n$ classes of insurance business, the distribution of the number of the ruined business classes in a finite time interval and the Laplace transform of the ruin time of the correlated risk process are investigated. Some closed form results are obtained. Numerical examples are presented to explain how the collection of insurance risk increases the solvency of an insurer.
\end{abstract}

Keywords: correlated risk model; Cox process; joint ruin probability; modified Bessel function; multi-dimensional risk models; regime switching; time of ruin; upper bounds

* Corresponding author: u3001202@hku.hk 


\section{Introduction}

Ruin theory for correlated risk models and multi-dimensional risk models are interesting topics both in insurance mathematics and in practice. There are different ways to construct the dependence among the classes of insurance business. One of them is the so-called common shock approach which shows that an external event can simultaneously cause claims in different classes of business. See, for example, Ambagaspitiya (2003), Lindskog and McNeil (2003), and Wang and Yuen (2005) for details.

Chan et al. (2003) and Dang et al. (2009) investigate the joint ruin probabilities under some two-dimensional insurance risk models with common shock. Such a multidimensional risk model can be regarded as a vector process, in which the component processes are correlated. We can obtain a correlated risk process by summing up all component processes of the vector process. It is usually difficult to derive explicit results for the joint ruin probabilities in multi-dimensional risk models. It is of interest to consider various different multi-dimensional risk models that could capture and measure certain features of correlated insurance risk.

Insurance risk models with regime switching have been widely studied. In this kind of models, the intensities of claims occurring are assumed to be affected by the state of environment. See, for example, Asmussen and Albrecher (2010), Albrecher and Boxma (2005), Lu and Li (2005), and Ng and Yang (2006). In this paper, we introduce regime switching to a multi-dimensional compound Cox risk model with conditional independence. Since the intensity of the Cox process represents the rate of claims occurring and it is stochastic, Eq. (3) in Chapter 2 of Grandell (1991, p. 36) shows that the interesting problem for a Compound Cox risk model is usually its finite time ruin probability. We investigate the finite time ruin probability and the joint finite time ruin probability for the proposed multi-dimensional compound Cox risk process in this paper.

One of the interesting problems for a correlated risk model is to analyze how the dependence among the classes of business impacts the insurance risk. This can help us to effectively manage the correlated insurance risk. For example, we can apply the ruin theory for a correlated risk process to explain how the collection of the insurance risks increases the solvency of an insurer and to prepare the initial reserve for a given solvency level. We will show these by numerical results for the proposed correlated risk model.

We consider an insurer with $n(n \geq 2)$ dependent classes of insurance business. The surplus process, $\left\{R_{j}(t)\right\}$, of the $j$ th class of business is defined as

$$
R_{j}(t)=u_{j}+\int_{0}^{t} c_{j}(s) d s-\sum_{k=1}^{N_{j}(t)} Z_{k}^{(j)}, \text { for } j=1, \cdots, n
$$

where the constant $u_{j} \geq 0$ is the initial reserve of the $j$ th class of business, $c_{j}(t)$ is the rate of premium income from the $j$ th class of business at time $t, N_{j}(t)$ is the number 
of the claims occurring in $[0, t]$ and $Z_{k}^{(j)}$ is the size of the $k$ th claim in the $j$ th class of business.

Following Wang and Yuen (2005), we define the claim number processes $\left\{N_{j}(t)\right\}$ as follows. We assume that stochastic sources that may cause a claim in at least one of the $n$ classes are classified into $m$ groups. We denote the numbers of the events occurring in the $m$ groups by $m$ Cox processes, $\left\{N^{1}(t)\right\}, \cdots,\left\{N^{m}(t)\right\}$ with intensity processes $\left\{\bar{\lambda}_{1}(t)\right\}, \cdots,\left\{\bar{\lambda}_{m}(t)\right\}$, respectively. This means that for each $\left\{N^{i}(t)\right\}$, there exists a standard Poisson process $\left\{\bar{N}^{i}(t)\right\}$, which is independent of $\left\{\bar{\lambda}_{i}(t)\right\}$, such that

$$
N^{i}(t)=\bar{N}^{i}\left(\bar{\Lambda}_{i}(t)\right) \text { with } \bar{\Lambda}_{i}(t)=\int_{0}^{t} \bar{\lambda}_{i}(s) d s, i=1, \cdots, m,
$$

see Grandell (1991). We suppose that each event in the $i$ th source may cause a claim in the $j$ th class of business with a constant probability $p_{i j}$ and that whether or not an event in the $i$ th source cause a claim in the $j$ th class is independent of all other events for $i=1, \cdots, m$ and $j=1, \cdots, n$. Let $N_{i j}(t)$ denote the number of claims occurring in the $j$ th class generated from the events in the $i$ th group up to time $t$. Thus, we have

$$
N_{j}(t)=\sum_{i=1}^{m} N_{i j}(t), t \geq 0
$$

Assumption 1.1: $\left\{\bar{N}^{1}(t)\right\}, \cdots,\left\{\bar{N}^{m}(t)\right\}$ are independent standard Poisson processes and independent of all intensity processes.

Assumption 1.2: $\left\{Z_{k}^{(1)}\right\}, \cdots,\left\{Z_{k}^{(n)}\right\}$ are $n$ independent i.i.d. series and independent of all claim number processes.

Define $G_{t}=\sigma\left\{\bar{\lambda}_{i}(s), s \leq t, i=1, \cdots, m\right\}, t \geq 0$. Denote the common distribution function of $\left\{Z_{n}^{(j)}\right\}$ by $F_{j}(x)$.

Remark 1.1: Under Assumptions 1.1 and 1.2, we have

(1) The claim number process $\left\{N_{i j}(t)\right\}$ is a Cox process with intensity process $\lambda_{i j}(t)=$ $p_{i j} \bar{\lambda}_{i}(t), t \geq 0$ for $i=1, \cdots, m$ and $j=1, \cdots, n$;

(2) The claim number process $\left\{N_{j}(t)\right\}$ is a Cox process with accumulated intensity process

$\Lambda_{j}(t)=\int_{0}^{t} \lambda_{j}(s) d s=\sum_{i=1}^{m} p_{i j} \bar{\Lambda}_{i}(t)$ with $\lambda_{j}(t)=\sum_{i=1}^{m} p_{i j} \bar{\lambda}_{i}(t), t \geq 0$ for $j=1, \cdots, n$;

(3) The $m$ vector processes $\left\{N^{1}(t), N_{11}(t), \cdots, N_{1 n}(t)\right\},\left\{N^{2}(t), N_{21}(t), \cdots, N_{2 n}(t)\right\}$, $\cdots,\left\{N^{m}(t), N_{m 1}(t), \cdots, N_{m n}(t)\right\}$ are conditionally independent, given $G_{t}$.

(4) The claim number processes $\left\{N_{i 1}(t)\right\}, \cdots,\left\{N_{i n}(t)\right\}$ are conditionally independent, given $N^{i}(t)$ and $G_{t}$ for $t \geq 0$ and $i=1, \cdots, m$.

We now assume that all intensity processes of claim number processes depend on the state of environment. Following Asmussen and Albrecher (2010), Lin et al. (2009) 
and Elliott et al. (2005), we denote the state of environment by a time-homogeneous Markov chain which is denoted by $\{X(t), t \geq 0\}$. For convenience, the state space of $\{X(t)\}$ is chosen as $D=\left\{e_{1}, e_{2}, \ldots, e_{N}\right\}$ with $e_{i}=(0, \ldots, 0,1,0, \ldots, 0)^{\prime} \in R^{N}$. The process $\{X(t)\}$ has the representation (see Elliott et al. (1994)):

$$
X(t)=X(0)+\int_{0}^{t} \mathbf{A} X(u) d u+M(t),
$$

where $\mathbf{A}$ is the intensity matrix of Markov chain $\{X(t)\}$ given by

$$
\mathbf{A}=\left(\begin{array}{llll}
-a_{11} & a_{12} & \cdots & a_{1 N} \\
\cdots & \cdots & \cdots & \cdots \\
a_{N 1} & a_{N 2} & \cdots & -a_{N N}
\end{array}\right)
$$

with all $a_{i j} \geq 0$ and $\{M(t), t \geq 0\}$ is a martingale with respect to the filtration $F_{t}^{X}=\sigma\{X(s), s \leq t\}$. Similar to Asmussen and Albrecher (2010) and Zhu and Yang (2008), we define

$$
\bar{\lambda}_{i}(t)=\lambda_{i l}, \text { if } X(t)=e_{l}, i=1, \cdots, m, l=1, \cdots, N,
$$

where $\lambda_{i l}$ are nonnegative constants. This means that the intensity processes of $\left\{N^{1}(t)\right\},\left\{N^{2}(t)\right\}, \cdots,\left\{N^{m}(t)\right\}$ are functionals of Markov chain $\{X(t)\}$. It is easy to see that $F_{t}^{X}=G_{t}$ for $t \geq 0$ under definition (1.6).

There are some other different choices for the intensity processes in the compound Cox risk models. See, for example, Grandell (1991) and Albrecher and Asmussen (2006).

Assumption 1.3: $\lambda_{i j}>0,\left(p_{i 1}, \cdots, p_{\text {in }}\right) \neq 0$, for $i=1, \cdots, m$, and $j=1, \cdots, N$.

Write $u=\sum_{j=1}^{n} u_{j}$. Set

$$
C(t)=\sum_{j=1}^{n} \int_{0}^{t} c_{j}(s) d s \text { and } S(t)=\sum_{j=1}^{n} \sum_{k=1}^{N_{j}(t)} Z_{k}^{(j)} .
$$

Then, the surplus process of the insurer is

$$
R(t)=\sum_{j=1}^{n} R_{j}(t)=u+C(t)-S(t), t \geq 0 .
$$

The surplus process $\{R(t)\}$ defined by (1.7) is a sum of $n$ risk processes which are correlated. We call it a correlated risk process.

In Section 2, we derive upper bounds for the ruin probability and some relationships among the Lundberg exponents of various risk models in this paper under certain assumptions. In Section 3, we present a closed form formula for calculating the joint finite time ruin probability for the proposed multi-dimensional compound Cox risk processes $\left\{R_{j}(t)\right\}$ 's via the modified Bessel function and the finite time ruin probability of classical risk model. In Section 4, we obtain a closed form formula for calculating the Laplace transform of ruin time for the proposed correlated risk process. Some numerical results for finite time ruin probabilities are presented in Section 5. 


\section{Upper bounds for ruin probability}

Under Assumptions 1.1 and 1.2, the following lemma is easy to see.

Lemma 2.1: Let $0<t_{1}<t_{2}, n_{i j} \leq n_{i}, n_{i j}, n_{i} \in\{0,1,2, \cdots\}$, we have

$$
P\left(N_{i j}\left(t_{1}\right)=n_{i j} \mid N^{i}\left(t_{2}\right)=n_{i}, G_{t}\right)=C_{n_{i}}^{n_{i j}}\left(p_{i j} \frac{t_{1}}{t_{2}}\right)^{n_{i j}}\left(1-p_{i j} \frac{t_{1}}{t_{2}}\right)^{n_{i}-n_{i j}}
$$

and

$$
\begin{aligned}
& P\left(N^{i}(t)=n_{i}, N_{i j}(t)=n_{i j}, j=1, \cdots, n \mid G_{t}\right) \\
= & \frac{\left(\bar{\Lambda}_{i}(t)\right)^{n_{i}}}{n_{i} !} e^{-\bar{\Lambda}_{i}(t)} \prod_{j=1}^{n} C_{n_{i}}^{n_{i j}}\left(p_{i j}\right)^{n_{i j}}\left(1-p_{i j}\right)^{n_{i}-n_{i j}}
\end{aligned}
$$

for $i=1, \cdots, m$.

Define

$$
M_{j}(r)=E\left[e^{r Z_{k}^{(j)}}\right], j=1, \cdots, n
$$

and

$$
\Pi_{i}(r)=\prod_{j=1}^{n}\left(p_{i j}\left(M_{j}(r)-1\right)+1\right)-1, i=1, \cdots, m .
$$

From Lemma 2.1 and following the idea to prove Eq.(2.3) in Wang and Yuen (2005), we obtain

$$
E\left[e^{r S(t)}\right]=E\left[e^{\sum_{i=1}^{m} \bar{\Lambda}_{i}(t) \Pi_{i}(r)}\right]
$$

From the assumption of conditional independence we have

$$
E\left[e^{r[S(t)-S(s)]}\right]=E\left[e^{\sum_{i=1}^{m}\left(\bar{\Lambda}_{i}(t)-\bar{\Lambda}_{i}(s)\right) \Pi_{i}(r)}\right] .
$$

For simplicity and without loss of generality, we assume that all the classes of business have the same net conditional profit coefficient $\theta>0$. Following Asmussen and Albrecher (2010) and Zhu and Yang (2008), we define the rate of premium income in the $j$ th class as

$$
c_{j}(t)=(1+\theta) \mu_{j} \sum_{i=1}^{m} p_{i j} \lambda_{i l}, \text { if } X(t)=e_{l}, \text { for } l=1, \cdots, N .
$$

From (2.7) we have

$$
C_{j}(t)=\int_{0}^{t} c_{j}(s) d s=(1+\theta) \mu_{j} \sum_{i=1}^{m} p_{i j} \bar{\Lambda}_{i}(t), j=1, \cdots, n,
$$


and

$$
C(t)=\sum_{j=1}^{n} C_{j}(t)=(1+\theta) \sum_{i=1}^{m} L_{i} \bar{\Lambda}_{i}(t)
$$

where

$$
L_{i}=\sum_{j=1}^{n} \mu_{j} p_{i j} \text { for } i=1, \cdots, m
$$

The following assumption is motivated from Assumption 4 of Grandell (1991, p. 2) which insure that the Lundberg exponents in Theorems 2.2 and 2.3 exist.

Assumption 2.1: There exist $r_{\infty}^{j}>0$ such that $M_{j}(r) \uparrow \infty$ when $r \uparrow r_{\infty}^{i}$ (we allow for the possibility of $\left.r_{\infty}^{i}=\infty\right)$ for $j=1, \cdots, n$.

Given a constant $k>0$, if we choose $F_{j}^{\prime}(z)=\frac{k}{z^{2}} e^{-z}$ for $z>1$, Assumption 2.1 will not hold any more. See Remark 5 of Grandell (1991, p. 3).

Theorem 2.1: Define a filtration $\mathbf{F}$ by $F_{t}=G_{\infty} \vee F_{t}^{R}$ with $F_{t}^{R}=\sigma\{R(s), 0 \leq s \leq t\}$ and process $\{\widetilde{M}(t)\}$ given by

$$
\widetilde{M}(t)=\frac{e^{-r R(t)}}{e^{A(t, r)}}
$$

where

$$
A(t, r)=\sum_{i=1}^{m} \bar{\Lambda}_{i}(t)\left[\Pi_{i}(r)-(1+\theta) L_{i} r\right]
$$

Then, $\{\widetilde{M}(t)\}$ is an $\mathbf{F}$-martingale.

Proof: By Eq. (2.5) and from the conditional independence assumptions we have $E^{F_{s}}[\widetilde{M}(t)]=E\left[\widetilde{M}(t) \mid F_{s}\right]=\widetilde{M}(s) E^{F_{s}}\left[\frac{e^{-r[R(t)-R(s)]}}{e^{A(t, r)-A(s, r)}}\right]=\widetilde{M}(s) E\left[\frac{e^{A(t, r)-A(s, r)}}{e^{A(t, r)-A(s, r)}}\right]=\widetilde{M}(s)$.

This ends the proof.

The time of ruin and the probability of ruin for surplus process $\{R(t)\}$ is defined as

$$
T=\inf \{t>0: R(t)<0\} \text { and } \Psi(u)=P(T<\infty),
$$

respectively. By martingale approach of Gerber (1973) and similar to inequality (41) of Grandell (1991, p. 94), we have

$$
P\left(T \leq t \mid F_{0}\right) \leq \frac{e^{-r u}}{E\left[\widetilde{M}(T) \mid T \leq t, F_{0}\right]}
$$


Note that

$$
E\left[\widetilde{M}(T) \mid T \leq t, F_{0}\right] \geq \inf _{0 \leq s \leq t} e^{-A(s, r)}
$$

It follows that

$$
P(T \leq t) \leq e^{-r u} E\left[\sup _{0 \leq s \leq t} e^{A(s, r)}\right]
$$

Write

$$
H(r)=E\left[\sup _{t \geq 0} e^{A(t, r)}\right] .
$$

From (2.13) we have

$$
\Psi(u) \leq H(r) e^{-r u}
$$

The Lundberg exponent $R$ for surplus process $\{R(t)\}$ is defined by

$$
R=\sup \{r>0: H(r)<\infty\} \text {. }
$$

From (2.14) we have the following theorem, which corresponds to Theorem 20 in Chapter 4 of Grandell (1991).

Theorem 2.2: For every $\varepsilon>0$ such that $0<\varepsilon<R$, under Assumptions 1.3 and 2.1 we have

$$
\Psi(u) \leq H(R-\varepsilon) e^{-(R-\varepsilon) u}
$$

where $H(R-\varepsilon)<\infty$.

Proof: Under Assumption 1.2, we see that all $\bar{A}_{i}(t)>0$ for $t>0$ and $\bar{A}_{i}(t) \rightarrow \infty$ as $t \rightarrow$ $\infty$. Under Assumption 2.1, $A(t, r)$ is a convex function of variable $r$ on $\left[0, \min \left\{r_{j}^{\infty}, j=\right.\right.$ $1, \cdots, n\}), A(t, 0)=0$, and there exists a sufficient small positive number $\varepsilon>0$ such that $A(t, r)$ is less than zero in the interval $(0, \varepsilon)$. Therefore, the Lundberg exponent $R>0$ exists. Assumption 2.1 implies that $R<\min \left\{r_{j}^{\infty}, j=1, \cdots, n\right\}$. Thus, the inequality (2.15) holds true.

The time of ruin and the probability of ruin for surplus process $\left\{R_{j}(t)\right\}$ is defined as

$$
T_{j}=\inf \left\{t>0: R_{j}(t)<0\right\} \text { and } \Psi_{j}\left(u_{j}\right)=P\left(T_{j}<\infty\right) \text {, }
$$

respectively. Write

$$
H_{j}(r)=E\left[\sup _{t \geq 0} e^{A_{j}(t, r)}\right],
$$


where

$$
A_{j}(t, r)=\left[\left(M_{j}(r)-1\right)-(1+\theta) \mu_{j} r\right] \sum_{i=1}^{m} \bar{\Lambda}_{i}(t) p_{i j} .
$$

Similar to (2.14) we have

$$
\Psi_{j}\left(u_{j}\right) \leq H_{j}(r) e^{-r u_{j}}
$$

Define

$$
g_{j}(r)=M_{j}(r)-1-(1+\theta) \mu_{j} r .
$$

It is easy to see that $g_{j}(r)$ is a convex function of variable $r$ on $\left(0, r_{j}^{\infty}\right)$ and has a unique positive root $R_{j}$ such that $0<R_{j}<r_{j}^{\infty}$. We conclude that $H_{j}(r)=\infty$ for all $r>R_{j}$ and $H_{j}\left(R_{j}\right)=1$. Therefore, we have the following theorem.

Theorem 2.3: Under Assumptions 1.3 and 2.1 we have

$$
\Psi_{j}\left(u_{j}\right) \leq e^{-R_{j} u_{j}} \text { for } j=1, \cdots, n .
$$

We call the unique positive root $R_{j}$ of equation $g_{j}(r)=0$ the Lundberg exponent for surplus process $\left\{R_{j}(t)\right\}$.

The following theorem presents the relationship between $R$ and $R_{j}$ 's.

Theorem 2.4: Under Assumptions 1.3 and 2.1 we have

$$
R \leq \max \left\{R_{1}, \cdots, R_{n}\right\} .
$$

Proof: Under Assumptions 1.3 and 2.1, we see that the Lundberg exponents $R, R_{1}, \cdots, R_{n}$ are all finite. Note that,

$$
\sum_{j=1}^{n} p_{i j}\left(M_{j}(r)-1\right) \leq \Pi_{i}(r)=\prod_{j=1}^{n}\left(p_{i j}\left(M_{j}(r)-1\right)+1\right)-1 .
$$

It follows that

$$
\begin{aligned}
& A(t, r) \\
= & \sum_{i=1}^{m} \bar{\Lambda}_{i}(t)\left[\Pi_{i}(r)-(1+\theta) L_{i} r\right] \\
\geq & \sum_{i=1}^{m} \bar{\Lambda}_{i}(t)\left[\sum_{j=1}^{n} p_{i j}\left(M_{j}(r)-1\right)-(1+\theta) r \sum_{j=1}^{n} p_{i j} \mu_{j}\right] \\
= & \sum_{j=1}^{n} A_{j}(t, r) .
\end{aligned}
$$


Suppose

$$
R>\max \left\{R_{1}, \cdots, R_{n}\right\} .
$$

Then, there exists a sufficient small positive number $\varepsilon$ such that

$$
R-\varepsilon>\max \left\{R_{1}, \cdots, R_{n}\right\} .
$$

Since all $A_{j}(t, R-\varepsilon)$ are positive, it follows from (2.22) that

$$
A(t, R-\varepsilon) \geq \sum_{j=1}^{n} A_{j}(t, R-\varepsilon)>A_{j}(t, R-\varepsilon) .
$$

Eq. (2.23) implies that

$$
\infty>H(R-\varepsilon)=E\left[\sup _{t \geq 0} e^{A(t, R-\varepsilon)}\right] \geq E\left[\sup _{t \geq 0} e^{A_{j}(t, R-\varepsilon)}\right]=\infty .
$$

This contradiction proves the result.

\section{Conditional independence and joint survival prob- ability}

In this section, we consider the calculation of the joint ruin probability for the $n$ dimensional risk models $\left\{R_{1}(t), \cdots, R_{n}(t)\right\}$. Define

$$
\bar{R}_{j}(t)=u_{j}+(1+\theta) \mu_{j} t-\sum_{k=1}^{\bar{N}_{j}(t)} Z_{k}^{(j)}, t \geq 0 .
$$

Then $\left\{\bar{R}_{j}(t)\right\}$ is a compound Poisson risk process. We have $R_{j}(t)=\bar{R}_{j}\left(\Lambda_{j}(t)\right)$.

The times of ruin for risk processes $\left\{\bar{R}_{j}(t)\right\}$ is defined as $\bar{T}_{j}=\inf \left\{t>0: \bar{R}_{j}(t)<0\right\}$. It is easy to see that

$$
\begin{aligned}
& P\left(T_{j}>t \mid G_{t}\right)=P\left(\inf _{0 \leq s \leq t} R_{j}(s) \geq 0 \mid G_{t}\right) \\
& =P\left(\inf _{0 \leq s \leq \Lambda_{j}(t)} \bar{R}_{j}(s) \geq 0 \mid G_{t}\right)=P\left(\bar{T}_{j}>\Lambda_{j}(t) \mid G_{t}\right) .
\end{aligned}
$$

Similarly, for any subset $Q \subset\{1,2, \cdots, n\}$ we have

$$
P\left(\bigcap_{j \in Q}\left(T_{j}>t\right) \mid G_{t}\right)=P\left(\bigcap_{j \in Q}\left(\bar{T}_{j}>\Lambda_{j}(t)\right) \mid G_{t}\right) .
$$


Thus, the joint survival probability for risk processes $\left\{R_{j}(t)\right\}, j \in Q$, is

$$
\begin{aligned}
& P\left(\bigcap_{j \in Q}\left(T_{j}>t\right)\right)=E\left[P\left(\bigcap_{j \in Q}\left(T_{j}>t\right) \mid G_{t}\right)\right] \\
& =E\left[P\left(\bigcap_{j \in Q}\left(\bar{T}_{j}>\Lambda_{j}(t)\right) \mid G_{t}\right)\right] \\
& =P\left(\bigcap_{j \in Q}\left(\bar{T}_{j}>\Lambda_{j}(t)\right)\right) .
\end{aligned}
$$

The following assumption presents sufficient conditions under which we are able to derive some closed form expressions for the joint ruin probability for surplus processes $R_{j}(t)$ 's.

Assumption 3.1: Assume that $m=n, p_{i i} \neq 0$ for $i=1, \cdots, n$ and $p_{i j}=0$ for $i \neq j$.

Remark 3.1: Under Assumption 3.1, we see that process $\left\{N_{j}(t)\right\}$ is the $p_{j j}$-thinning of Cox process $\left\{N^{j}(t)\right\}$. From this, together with Assumptions 1.1-1.3, we see that processes $R_{j}(t)$ 's are correlated but conditionally independent, given the historical information of process $\{X(t)\}$.

Under Assumption 3.1 and from Eq. (3.3), we can verify that the following theorem holds true.

Theorem 3.1: Under Assumption 3.1, for any subset $Q \subset\{1,2, \cdots, n\}$ we have

$$
P\left(\bigcap_{j \in Q}\left(T_{j}>t\right)\right)=E\left[\prod_{j \in Q} P\left(\bar{T}_{j}>\Lambda_{j}(t) \mid G_{t}\right)\right] .
$$

Note that Assumption 1.3 implies that $\Lambda_{j}(t) \uparrow \infty(t \uparrow \infty)$. Then, from Eq. (3.3) we obtain

$$
P\left(\bigcap_{j=1}^{n}\left\{T_{j}=\infty\right\}\right)=\prod_{j=1}^{n} P\left(\bar{T}_{j}=\infty\right) .
$$

In the case that all $u_{j}=0$ we have $\left.P\left(\bar{T}_{j}=\infty\right)\right|_{u_{j}=0}=\frac{1}{1+\theta}$, see Gerber (1979) and Grandell (1991). Therefore, from (3.5) we obtain

$$
\left.P\left(\bigcap_{j=1}^{n}\left\{T_{j}=\infty\right\}\right)\right|_{u_{j}=0, j=1, \cdots, n}=\frac{1}{(1+\theta)^{n}} .
$$

When $F_{j}(z)=1-e^{-\beta_{j} z}$ for $z>0$ and $j=1, \cdots, n$, from (3.6) and Eq. (II) of Grandell (1991, p. 6) we obtain

$$
P\left(\bigcap_{j=1}^{n}\left\{T_{j}=\infty\right\}\right)=\prod_{j=1}^{n}\left[1-\frac{1}{(1+\theta)} e^{-\frac{\theta \beta_{j} u_{j}}{1+\theta}}\right] .
$$


Another interesting distribution related to the multi-dimensional risk model is the number of the classes of business that ruin in the finite time interval $[0, t]$. Denoted by $N_{t}$ the number of the classes of business that ruin up to time $t$. Similar to Giesecke (2003) (see also Liang and Wang (2012)) we have

$$
\begin{aligned}
& P\left(N_{t}=k\right) \\
= & P\left(\sum_{j=1}^{n} I_{\left(T_{j}>t\right)}=n-k\right) \\
= & \sum_{i=n-k}^{n}\left(\begin{array}{l}
i \\
n-k
\end{array}\right)(-1)^{i-(n-k)} \sum_{Q \subset\{1, \cdots, n\},|Q|=i} P\left(\bigcap_{j \in Q}\left\{T_{j}>t\right\}\right) \\
= & \sum_{i=n-k}^{n}\left(\begin{array}{l}
i \\
n-k
\end{array}\right)(-1)^{i-(n-k)} \sum_{Q \subset\{1, \cdots, n\},|Q|=i} \\
& P\left(\bigcap_{j \in Q}\left\{\bar{T}_{j}>\Lambda_{j}(t)\right\}\right), k=0, \cdots, n-1,
\end{aligned}
$$

and

$$
P\left(N_{t}=n\right)=1-\sum_{i=0}^{n-1} P\left(N_{t}=i\right)
$$

where $|Q|$ denote the number of the elements of set $Q$. It is easy to see that

$$
P\left(N_{\infty}=0\right)=P\left(\bigcap_{j=1}^{n}\left\{T_{j}=\infty\right\}\right)=\prod_{j=1}^{n} P\left(\bar{T}_{j}=\infty\right) .
$$

It follows from (3.5) and (3.6) that

$$
\left.P\left(N_{\infty}=0\right)\right|_{u_{j}=0, j=1, \cdots, n}=\frac{1}{(1+\theta)^{n}} .
$$

Similar to (3.7), we have

$$
P\left(N_{\infty}=0\right)=\prod_{j=1}^{n}\left[1-\frac{1}{(1+\theta)} e^{-\frac{\theta \beta_{j} u_{j}}{1+\theta}}\right]
$$

when $F_{j}(z)=1-e^{-\beta_{j} z}$ for $z>0$ and $j=1, \cdots, n$. For $k>0$, we have

$$
\begin{aligned}
& \left.P\left(N_{\infty}=k\right)\right|_{u_{j}=0, j=1, \cdots, n} \\
= & \sum_{i=n-k}^{n}\left(\begin{array}{l}
i \\
n-k
\end{array}\right)(-1)^{i-(n-k)}\left(\begin{array}{l}
n \\
i
\end{array}\right) \frac{1}{(1+\theta)^{i}} .
\end{aligned}
$$

The distribution of the occupation time of a Markov chain is helpful for us to calculate the joint ruin probability. To show this and to present some closed form expressions, 
we consider the Markov chain $\{X(t)\}$ having only two states. In this case, its intensity matrix can be written as

$$
\mathbf{A}=\left(\begin{array}{ll}
-a_{11} & a_{11} \\
a_{22} & -a_{22}
\end{array}\right)
$$

Let $O_{l}(t)$ denote the occupation time of Markov chain $X(t)$ spending at the state $e_{l}$ up to time $t$. That is,

$$
O_{l}(t)=\int_{0}^{t} I\left(X(s)=e_{l}\right) d s, l=1,2 .
$$

From Eq. (3.15) we have

$$
\Lambda_{j}(t)=p_{j j}\left[\left(\lambda_{j 1}-\lambda_{j 2}\right) O_{1}(t)+\lambda_{j 2} t\right], j=1, \cdots, n .
$$

Given $X(0)=e_{1}$, Kovchegov et al. (2010, p. 106) show that $O_{1}(t)$ has density function $f_{1}(t, x)$ given by

$$
\begin{aligned}
f_{1}(t, x)= & e^{-a_{11} t} \delta_{t}(x)+e^{-a_{11} x} e^{-a_{22}(t-x)}\left[a_{11} I_{0}\left(2 \sqrt{a_{11} a_{22} x(t-x)}\right)\right. \\
& \left.+\sqrt{\frac{a_{11} a_{22} x}{t-x}} I_{1}\left(2 \sqrt{a_{11} a_{22} x(t-x)}\right)\right]
\end{aligned}
$$

for $0 \leq x \leq t$, where

$$
I_{\rho}(z)=\sum_{k=0}^{\infty} \frac{1}{k ! \Gamma(k+\rho+1)}\left(\frac{z}{2}\right)^{2 k+\rho}, \rho>-1,
$$

is the modified Bessel functions. For any $Q \subset\{1, \cdots, n\}$, from Eq. (3.17) and Theorem 4.1 we see that

$$
\begin{aligned}
& P\left(\bigcap_{j \in Q}\left(T_{j}>t\right)\right) \\
= & E\left[\prod_{j \in Q}\left[1-P\left(\bar{T}_{j} \leq \Lambda_{j}(t) \mid G_{t}\right)\right]\right] \\
= & E\left[\prod_{j \in Q}\left[1-P\left(\bar{T}_{j} \leq p_{j j}\left(\left(\lambda_{j 1}-\lambda_{j 2}\right) O_{1}(t)+\lambda_{j 2} t\right) \mid O_{1}(t), G_{t}\right)\right]\right] \\
= & E\left[\prod_{j \in Q}\left[1-P\left(\bar{T}_{j} \leq p_{j j}\left(\left(\lambda_{j 1}-\lambda_{j 2}\right) O_{1}(t)+\lambda_{j 2} t\right)\right)\right]\right] \\
= & \int_{0}^{t}\left(\prod_{j \in Q}\left[1-P\left(\bar{T}_{j} \leq p_{j j}\left(\left(\lambda_{j 1}-\lambda_{j 2}\right) s+\lambda_{j 2} t\right)\right)\right]\right) f_{1}(t, s) d s .
\end{aligned}
$$

Inserting Eq. (3.19) into Eq. (3.8) we obtain the following theorem which gives a closed form result for calculating the distribution of $N_{t}$ via the distributions of ruin times $\bar{T}_{j}$ 's and the distribution of $O_{l}(t)$. 
Theorem 3.2: Given $X(0)=e_{1}$ and let $0 \leq k \leq n-1$. Under Assumption 3.1 we have

$$
\begin{aligned}
& P\left(N_{t}=k\right) \\
= & \sum_{i=n-k}^{n}\left(\begin{array}{c}
i \\
n-k
\end{array}\right)(-1)^{i-(n-k)} \sum_{Q \subset\{1, \cdots, n\},|Q|=i} \\
& \int_{0}^{t}\left(\prod_{j \in Q}\left[1-P\left(\bar{T}_{j} \leq p_{j j}\left(\left(\lambda_{j 1}-\lambda_{j 2}\right) s+\lambda_{j 2} t\right)\right)\right]\right) f_{1}(t, s) d s .
\end{aligned}
$$

Theorem 3.2 and Eq. (3.19) show that the finite time ruin probability of classical risk processes (3.1) plays an important role for calculating the joint finite time ruin probability of multi-dimensional model. Proposition 1.3 of Asmussen and Albrecher (2010, p. 118) presents a closed form solution for the finite time ruin probability when the claim sizes are exponentially distributed. From Theorem 3.2 and Proposition 1.3 of Asmussen and Albrecher (2010, p. 118), we are able to obtain some closed form solutions for the joint survival probability for risk processes $\left\{R_{j}(t)\right\}, j \in Q$, and the distribution of $N_{t}$.

Corollary 3.1: Given $X(0)=e_{1}$, let $0 \leq k \leq n-1$ and $F_{j}(z)=1-e^{-\beta_{j} z}$ for $z>0$ and $j=1, \cdots, n$. Define

$$
\gamma=\frac{1}{1+\theta}, \zeta_{j}(s)=(1+\theta) p_{j j}\left(\left(\lambda_{j 1}-\lambda_{j 2}\right) s+\lambda_{j 2} t\right)
$$

Under Assumption 3.1 we have

$$
\begin{aligned}
& P\left(\bigcap_{j \in Q}\left(T_{j}>t\right)\right) \\
= & \int_{0}^{t}\left(\prod_{j \in Q}\left[1-\gamma e^{-(1-\gamma) \beta_{j} u_{j}}+\frac{1}{\pi} \int_{0}^{\pi} \frac{f_{j 1}(x, s) f_{j 2}(x)}{f_{j 3}(x)} d x\right]\right) f_{1}(t, s) d s
\end{aligned}
$$

and

$$
\begin{aligned}
& P\left(N_{t}=k\right) \\
= & \sum_{i=n-k}^{n}\left(\begin{array}{c}
i \\
n-k
\end{array}\right)(-1)^{i-(n-k)} \sum_{Q \subset\{1, \cdots, n\},|Q|=i} \\
& \int_{0}^{t}\left(\prod_{j \in Q}\left[1-\gamma e^{-(1-\gamma) \beta_{j} u_{j}}+\frac{1}{\pi} \int_{0}^{\pi} \frac{f_{j 1}(x, s) f_{j 2}(x)}{f_{j 3}(x)} d x\right]\right) f_{1}(t, s) d s,
\end{aligned}
$$

where

$$
\begin{aligned}
& f_{j 1}(x, s)=\gamma e^{2 \sqrt{\gamma} \zeta_{j}(s) \cos x-(1+\gamma) \zeta_{j}(s)+\beta_{j} u_{j}(\sqrt{\gamma} \cos x-1)}, \\
& f_{j 2}(x)=\cos \left(\beta_{j} u_{j} \sqrt{\gamma} \sin x\right)-\cos \left(\beta_{j} u_{j} \sqrt{\gamma} \sin x+2 x\right), \\
& f_{j 3}(x)=1+\gamma-2 \sqrt{\gamma} \cos x .
\end{aligned}
$$




\section{Laplace transform for ruin time $T$}

To calculate the finite time ruin probability for the correlated risk model $\{R(t)\}$ under Assumption 3.1, we consider the Laplace transform of the ruin time. For simplicity, we consider the case with $n=m=N=2$, where the intensity matrix of $\{X(t)\}$ is given by Eq. (3.14). In this case, we have

$$
R(t)=u+(1+\theta)\left(\mu_{1} p_{11} \bar{\Lambda}_{1}(t)+\mu_{2} p_{22} \bar{\Lambda}_{2}(t)\right)-\left(\sum_{k=1}^{N_{11}(t)} Z_{k}^{(1)}+\sum_{k=1}^{N_{22}(t)} Z_{k}^{(2)}\right)
$$

where $N_{11}(t)$ and $N_{22}(t)$ are correlated via Markov chain $\{X(t)\}$. The Laplace transform of the ruin time $T$ of $\{R(t)\}$ is defined as

$$
\begin{aligned}
L_{i}(u, \delta) & =E_{u, i}\left[e^{-\delta T} I(T<\infty)\right] \\
& =E\left[e^{-\delta T} I(T<\infty) \mid R(0)=u, X(0)=e_{i}\right], \text { for } i=1,2 .
\end{aligned}
$$

Similarly, we introduce notation $P_{u, i}$,

$$
P_{u, i}(T \leq t)=E\left[I\{T \leq t\} \mid R(0)=u, X(0)=e_{i}\right], i=1,2 .
$$

Write $\frac{\partial f(u, \delta)}{\partial u}$ as $f^{\prime}(u, \delta)$. We define operators $\mathcal{A}_{1}$ and $\mathcal{A}_{2}$ by

$$
\begin{aligned}
\mathcal{A}_{1} f(u, \delta)= & \alpha_{1} f(u, \delta)-\alpha_{2} f^{\prime}(u, \delta)-\lambda_{11} p_{11} \int_{0}^{\infty} f(u-z, \delta) d F_{1}(z) \\
& -\lambda_{21} p_{22} \int_{0}^{\infty} f(u-z, \delta) d F_{2}(z)
\end{aligned}
$$

and

$$
\begin{aligned}
\mathcal{A}_{2} f(u, \delta)= & \alpha_{3} f(u, \delta)-\alpha_{4} f^{\prime}(u, \delta)-\lambda_{12} p_{11} \int_{0}^{\infty} f(u-z, \delta) d F_{1}(z) \\
& -\lambda_{22} p_{22} \int_{0}^{\infty} f(u-z, \delta) d F_{2}(z)
\end{aligned}
$$

for $u>0$, respectively, where

$$
\begin{aligned}
& \alpha_{1}=a_{11}+\delta+\lambda_{11} p_{11}+\lambda_{21} p_{22}, \\
& \alpha_{2}=(1+\theta)\left(\mu_{1} \lambda_{11} p_{11}+\mu_{2} \lambda_{21} p_{22}\right), \\
& \alpha_{3}=a_{22}+\delta+\lambda_{12} p_{11}+\lambda_{22} p_{22}, . \\
& \alpha_{4}=(1+\theta)\left(\mu_{1} \lambda_{12} p_{11}+\mu_{2} \lambda_{22} p_{22}\right) .
\end{aligned}
$$

Theorem 4.1: The Laplace transforms of ruin time $T$ satisfy the following integrodifferential equations

$$
\begin{aligned}
& \mathcal{A}_{1} L_{1}(u, \delta)=a_{11} L_{2}(u, \delta), u>0 \\
& \mathcal{A}_{2} L_{2}(u, \delta)=a_{22} L_{1}(u, \delta), u>0 .
\end{aligned}
$$


Proof : We only prove Eq. (4.5). Eq. (4.6) can be proved similarly. Let $\tau_{1}$ and $\tau_{2}$ denote the first jump times of $\left\{N_{11}(t)\right\}$ and $\left\{N_{22}(t)\right\}$, respectively. Put $\tau_{0}=\tau_{1} \wedge \tau_{2} \wedge t$. Note that $\tau_{1}$ and $\tau_{2}$ are correlated. It is easy to see that $R(t) \geq 0$ for $t \in\left(0, \tau_{0}\right), u \geq 0$. Therefore, $P\left(T \geq \tau_{0}\right)=1$. Then, it follows from the Markov property of vector process $\{R(t), X(t)\}$ that

$$
L_{1}(u, \delta)=E_{u, 1}\left[e^{-\delta \tau_{0}} L_{X\left(\tau_{0}\right)}\left(R\left(\tau_{0}\right), \delta\right)\right], u \geq 0 .
$$

Let $D_{i}$ denote the time that is from the beginning of Markov chain $\{X(t)\}$ staying at state $e_{i}$ till to leaving it, $i=1,2$. Then, $D_{i}$ is an exponential random variable with parameter $a_{i i}$. By the assumption of conditional dependence, we obtain

$$
\begin{aligned}
& P_{u, 1}\left(D_{1}>\tau_{0}, \tau_{1} \wedge \tau_{2}>t\right) \\
= & P_{u, 1}\left(D_{1}>t\right) E_{u, 1}\left[P_{u, 1}\left(\tau_{1}>t, \tau_{2}>t \mid D_{1}>t, G_{t}\right)\right] \\
= & e^{-a_{11} t} E_{u, 1}\left[\prod_{j=1}^{2} P_{u, 1}\left(\tau_{j}>t \mid D_{1}>t, G_{t}\right)\right] \\
= & e^{-a_{11} t} E_{u, 1}\left[e^{-\int_{0}^{t} p_{11} \lambda_{11} d s} e^{-\int_{0}^{t} p_{22} \lambda_{21} d s}\right] \\
= & e^{-a_{11} t}\left(1-\left(p_{11} \lambda_{11}+p_{22} \lambda_{21}\right) t\right)+o(t) .
\end{aligned}
$$

Similar to Eq. (4.8) we have

$$
\begin{aligned}
& P_{u, 1}\left(D_{1} \leq \tau_{0}, \tau_{1} \wedge \tau_{2}>t\right) \\
= & \left(1-e^{-a_{11} t}\right) E_{u, 1}\left[\prod_{j=1}^{2} P_{u, 1}\left(\tau_{j}>t \mid D_{1} \leq t, G_{t}\right)\right] \\
= & \left(1-e^{-a_{11} t}\right) E_{u, 1}\left[e^{-\int_{0}^{t} p_{11} \bar{\lambda}_{1} d s} e^{-\int_{0}^{t} p_{22} \bar{\lambda}_{2} d s} \mid D_{1} \leq t\right] \\
= & a_{11} t+o(t) .
\end{aligned}
$$

It follows from Eq. (4.8) that

$$
\begin{aligned}
& E_{u, 1}\left[e^{-\delta \tau_{0}} L_{X\left(\tau_{0}\right)}\left(R\left(\tau_{0}\right), \delta\right) I\left\{D_{1}>\tau_{0}, \tau_{1} \wedge \tau_{2}>t\right\}\right] \\
= & e^{-\delta t} E_{u, 1}\left[L_{1}(R(t), \delta) \mid D_{1}>t, \tau_{1} \wedge \tau_{2}>t\right] P_{u, 1}\left(D_{1}>t, \tau_{1} \wedge \tau_{2}>t\right) \\
= & e^{-\delta t} E_{u, 1}\left[L_{1}(R(t), \delta) \mid D_{1}>t, \tau_{1} \wedge \tau_{2}>t\right] P_{u, 1}\left(D_{1}>t, \tau_{1} \wedge \tau_{2}>t\right) \\
= & e^{-\left(\delta+a_{11}\right) t} L_{1}\left(u+(1+\theta)\left(\mu_{1} \lambda_{11} p_{11}+\mu_{2} \lambda_{21} p_{22}\right) t, \delta\right) \\
& \times\left(1-\left(\lambda_{11} p_{11}+\lambda_{21} p_{22}\right) t+o(t)\right) \\
= & \left(1-\left(a_{11}+\delta+\lambda_{11} p_{11}+\lambda_{21} p_{22}\right) t\right) L_{1}(u, \delta) \\
& +(1+\theta)\left(\mu_{1} \lambda_{11} p_{11}+\mu_{2} \lambda_{21} p_{22}\right) t L_{1}^{\prime}(u, \delta)+o(t) .
\end{aligned}
$$

From Eq. (4.9) we obtain

$$
\begin{aligned}
& E_{u, 1}\left[e^{-\delta \tau_{0}} L_{X\left(\tau_{0}\right)}\left(R\left(\tau_{0}\right), \delta\right) I\left\{D_{1} \leq \tau_{0}, \tau_{1} \wedge \tau_{2}>t\right\}\right] \\
= & e^{-\delta t}\left(1-e^{-a_{11} t}\right) E_{u, 1}\left[L_{2}(R(t), \delta) \mid D_{1} \leq t, \tau_{1} \wedge \tau_{2}>t\right] P_{u, 1}\left(\tau_{1} \wedge \tau_{2}>t\right) \\
= & a_{11} t L_{2}(u, \delta)+o(t) .
\end{aligned}
$$


Write $\left\{\tau_{1} \in(t, t+d t)\right\}$ as $\left\{\tau_{1} \in d t\right\}$ for $d t>0$. We have

$$
\begin{aligned}
& P_{u, 1}\left(D_{1}>t, \tau_{1} \in d t, \tau_{2}>t\right) \\
= & e^{-a_{11} t} E_{u, 1}\left[E_{u, 1}\left[I\left(\tau_{1} \in d t, \tau_{2}>t\right) \mid D_{1}>t, G_{t+d t}\right]\right] \\
= & p_{11} \lambda_{11} e^{-\left(a_{11}+p_{11} \lambda_{11}+p_{22} \lambda_{21}\right) t} d t .
\end{aligned}
$$

It follows that

$$
\begin{aligned}
& E_{u, 1}\left[e^{-\delta \tau_{0}} L_{X\left(\tau_{0}\right)}\left(R\left(\tau_{0}\right), \delta\right) I\left\{D_{1}>\tau_{0}, \tau_{1} \wedge \tau_{2}<t, \tau_{1}<\tau_{2}\right\}\right] \\
= & \int_{0}^{t} e^{-\delta s} E_{u, 1}\left[L_{1}(R(s), \delta) \mid D_{1}>s, \tau_{1}=s, \tau_{2}>s\right] P_{u, 1}\left(D_{1}>s, \tau_{1} \in d s, \tau_{2}>s\right) \\
= & \int_{0}^{t} E_{u, 1}\left[L_{1}\left(u+(1+\theta)\left(\left(\mu_{1} p_{11} \bar{\Lambda}_{1}(s)+\mu_{2} p_{22} \bar{\Lambda}_{2}(s)\right)\right)-Z_{k}^{(1)}, \delta\right)\right] \\
& \quad p_{11} \lambda_{11} e^{-\left(\delta+a_{11}+p_{11} \lambda_{11}+p_{22} \lambda_{21}\right) t} d s \\
= & \lambda_{11} p_{11} t \int_{0}^{\infty} L_{1}(u-z, \delta) d F_{1}(z)+o(t) .
\end{aligned}
$$

Similar to Eq. (4.13) we have

$$
\begin{aligned}
& E_{u, 1}\left[e^{-\delta \tau_{0}} L_{X\left(\tau_{0}\right)}\left(R\left(\tau_{0}\right), \delta\right) I\left\{D_{1}>\tau_{0}, \tau_{1} \wedge \tau_{2}<t, \tau_{2}<\tau_{1}\right\}\right] \\
= & \lambda_{21} p_{22} t \int_{0}^{\infty} L_{1}(u-z, \delta) d F_{2}(z)+o(t) .
\end{aligned}
$$

It is easy to verify that

$$
E_{u, 1}\left[e^{-\delta \tau_{0}} L_{X\left(\tau_{0}\right)}\left(R\left(\tau_{0}\right), \delta\right) I\left\{D_{1} \leq \tau_{0}, \tau_{1} \wedge \tau_{2}<t\right\}\right]=o(t) .
$$

From Eqs. (4.7), (4.10), (4.11), (4.13), (4.14) and (4.15) we obtain Eq. (4.5). This ends the proof.

Remark 4.1: Since $L_{j}(u, \delta)=1$ for $u<0$, we have

$$
\int_{0}^{\infty} L_{j}(u-z, \delta) d F_{j}(z)=\int_{0}^{u} L_{j}(u-z, \delta) d F_{j}(z)+\left(1-F_{j}(u)\right), j=1,2 .
$$

Example 4.1: In this example we will derive some closed form expressions for the Laplace transforms $L_{j}(u, \delta)$ when the claim size of the two classes of insurance business are exponentially distributed. We assume that $F_{1}(z)=1-e^{-\beta_{1} z}$ and $F_{2}(z)=1-e^{-\beta_{2} z}$ for $z>0$ with $\beta_{1}>\beta_{2}$. Let $\mathbf{I}$ denote the identity operator and $\mathbf{D}$ denote the differential operator. Define

$$
\begin{aligned}
& \Theta_{1}(\mathbf{D})=\left(\alpha_{1} \mathbf{I}-\alpha_{2} \mathbf{D}\right)\left(\mathbf{D}+\beta_{1} \mathbf{I}\right)\left(\mathbf{D}+\beta_{2} \mathbf{I}\right)-\beta_{1} \lambda_{11} p_{11}\left(\mathbf{D}+\beta_{2} \mathbf{I}\right)-\beta_{2} \lambda_{21} p_{22}\left(\mathbf{D}+\beta_{1} \mathbf{I}\right), \\
& \Theta_{2}(\mathbf{D})=\left(\alpha_{3} \mathbf{I}-\alpha_{4} \mathbf{D}\right)\left(\mathbf{D}+\beta_{1} \mathbf{I}\right)\left(\mathbf{D}+\beta_{2} \mathbf{I}\right)-\beta_{1} \lambda_{12} p_{11}\left(\mathbf{D}+\beta_{2} \mathbf{I}\right)-\beta_{2} \lambda_{22} p_{22}\left(\mathbf{D}+\beta_{1} \mathbf{I}\right) .
\end{aligned}
$$

Since Eqs. (4.5) and (4.6) hold true only for $u>0$. For convenience we extend the definitions of $\mathcal{A}_{1} L_{1}(u, \delta)$ and $\mathcal{A}_{2} L_{2}(u, \delta)$ to $u<0$,

$$
\mathcal{A}_{1} L_{1}(u, \delta)=a_{11} \text { and } \mathcal{A}_{2} L_{2}(u, \delta)=a_{22} \text { for } u<0 .
$$


Then, from Eqs. (4.5), (4.6) and (4.16) we conclude that the Laplace transforms $L_{j}(u, \delta), j=1,2$, satisfy the following integro-differential equations

$$
\begin{aligned}
& \mathcal{A}_{2}\left(\mathcal{A}_{1} L_{1}(u, \delta)\right)=a_{11} a_{22} L_{1}(u, \delta), u>0 \\
& \mathcal{A}_{1}\left(\mathcal{A}_{2} L_{2}(u, \delta)\right)=a_{11} a_{22} L_{2}(u, \delta), u>0 .
\end{aligned}
$$

It follows from Eq. (4.17) that

$$
\left(\mathbf{D}+\beta_{1} \mathbf{I}\right)^{2}\left(\mathbf{D}+\beta_{2} \mathbf{I}\right)^{2} \mathcal{A}_{2}\left(\mathcal{A}_{1} L_{1}(u, \delta)\right)=\Theta_{1}(\mathbf{D}) \Theta_{2}(\mathbf{D}) L_{1}(u, \delta) .
$$

Therefore, we have

$$
\Theta_{1}(\mathbf{D}) \Theta_{2}(\mathbf{D}) L_{1}(u, \delta)=a_{11} a_{22}\left(\mathbf{D}+\beta_{1} \mathbf{I}\right)^{2}\left(\mathbf{D}+\beta_{2} \mathbf{I}\right)^{2} L_{1}(u, \delta) .
$$

From Eq. (4.19) we see that the characteristic equation for solving $L_{1}(u, \delta)$ can be written as

$$
\Theta_{1}(r) \Theta_{2}(r)=a_{11} a_{22}
$$

where

$$
\begin{aligned}
& \Theta_{1}(r)=\alpha_{1}-\alpha_{2} r-\frac{\beta_{1} \lambda_{11} p_{11}}{r+\beta_{1}}-\frac{\beta_{2} \lambda_{21} p_{22}}{r+\beta_{2}}, \\
& \Theta_{2}(r)=\alpha_{3}-\alpha_{4} r-\frac{\beta_{1} \lambda_{12} p_{11}}{r+\beta_{1}}-\frac{\beta_{2} \lambda_{22} p_{22}}{r+\beta_{2}} .
\end{aligned}
$$

Note that $\Theta_{1}(0)>0, \Theta_{2}(0)>0$ and $\Theta_{1}(0) \Theta_{2}(0)-a_{11} a_{22}>0$. We can verify that Eq. (4.20) has six real roots $r_{i}$ 's with

$$
-\beta_{1}<r_{1}<r_{2}<-\beta_{2}<r_{3}<r_{4}<0<r_{5}<r_{6}<\infty .
$$

Note that $\lim _{u \rightarrow \infty} L_{1}(u, \delta)=0$. We see that $L_{1}(u, \delta)$ has the following form

$$
L_{1}(u, \delta)=\sum_{k=1}^{4} c_{k} e^{r_{k} u}, u \geq 0 .
$$

where $c_{k}$ 's are arbitrary constants.

Similarly, starting from Eq. (4.18) and following the same discussion as above we conclude that $L_{2}(u, \delta)$ has the following form

$$
L_{2}(u, \delta)=\sum_{k=1}^{4} d_{k} e^{r_{k} u}, u \geq 0 .
$$

where $d_{k}$ 's are arbitrary constants. 
Inserting $L_{1}(u, \delta)$ given in Eq. (4.21) into Eq. (4.17) and equating the coefficients of $u e^{-\beta_{i} u}$ and that of $e^{-\beta_{i} u}$ we obtain the system for determining the arbitrary constants $c_{k}$ 's

$$
\left\{\begin{array}{l}
\sum_{k=1}^{4} \frac{\beta_{1}}{r_{k}+\beta_{1}} c_{k}=1 \\
\sum_{k=1}^{4} \frac{\beta_{2}}{r_{k}+\beta_{2}} c_{k}=1, \\
\sum_{k=1}^{4} \frac{\beta_{1} \Theta_{1}\left(r_{k}\right)}{a_{11}\left(r_{k}+\beta_{1}\right)} c_{k}=1 \\
\sum_{k=1}^{4} \frac{\beta_{2} \Theta 1}{a_{11}\left(r_{k}+r_{k}\right)} c_{k}=1
\end{array}\right.
$$

Similar to (4.23) we have the following system for determining the arbitrary constants $d_{k}$ 's

$$
\left\{\begin{array}{l}
\sum_{k=1}^{4} \frac{\beta_{1}}{r_{k}+\beta_{1}} d_{k}=1, \\
\sum_{k=1}^{4} \frac{\beta_{2}}{r_{k}+\beta_{2}} d_{k}=1, \\
\sum_{k=1}^{4} \frac{\beta_{1} \Theta_{2}\left(r_{k}\right)}{a_{22}\left(r_{k}+\beta_{1}\right)} d_{k}=1 \\
\sum_{k=1}^{4} \frac{\beta_{2} \Theta_{2}\left(r_{k}\right)}{a_{22}\left(r_{k}+\beta_{2}\right)} d_{k}=1 .
\end{array}\right.
$$

\section{Numerical illustrations}

In this section, we present some numerical results for ruin probabilities under Assumption 3.1. For simplicity, we set $n=m=N=2$. This means that the insurer only has two classes of insurance business and the stochastic sources are classified into two groups.

Corollary 3.1 presents a method to calculate the joint survival probability numerically for risk processes $\left\{R_{j}(t)\right\}$ 's and the distribution of $N_{t}$ when the claim sizes are exponentially distributed. From Eq. (3.8) and Theorem 3.2 we have

$$
P\left(N_{t}=0\right)=P\left(\bar{T}_{1}>\Lambda_{1}(t), \bar{T}_{2}>\Lambda_{2}(t)\right)
$$

and

$$
P\left(N_{t}=1\right)=\sum_{j=1}^{2} P\left(\bar{T}_{j}>\Lambda_{j}(t)\right)-2 P\left(\bar{T}_{1}>\Lambda_{1}(t), \bar{T}_{2}>\Lambda_{2}(t)\right) .
$$

We will use the Gaver-Stehfest algorithm for inverting Laplace transform to calculate the distribution of ruin times for risk process (4.1) numerically. For nonnegative function $f(t)$, we denote by $\hat{F}$ its Laplace transform, that is,

$$
\hat{F}(\delta)=\int_{0}^{\infty} e^{-\delta t} f(t) \mathrm{d} t,
$$


where $\delta>0$. The Gaver-Stehfest algorithm for inverting Laplace transform is (see, for example, Kou and Wang (2003))

$$
f(t)=\lim _{m \rightarrow \infty} \sum_{k=1}^{m} \varpi(k, m) \bar{F}_{k}(t),
$$

where

$$
\varpi(k, m)=(-1)^{m-k} \frac{k^{m}}{k !(m-k) !}
$$

and

$$
\bar{F}_{m}(t)=\frac{\ln (2)}{t} \frac{(2 m) !}{m !(m-1) !} \sum_{k=0}^{m}(-1)^{k}\left(\begin{array}{l}
m \\
k
\end{array}\right) \hat{F}\left((m+k) \frac{\ln (2)}{t}\right) .
$$

Let $\hat{\psi}_{j}(u, \delta)$ denote the Laplace transform of $\psi_{j}(u, t)=\mathrm{P}_{u, j}(T \leq t)$. Similar to Kou and Wang (2003) and Dong et al. (2011) we have

$$
\left.\hat{\psi}_{j}(u, \delta)=\int_{0}^{\infty} e^{-\delta t} \mathrm{P}_{u, j}(T \leq t)\right) \mathrm{d} t=\frac{1}{\delta} \int_{0}^{\infty} e^{-\delta t} \mathrm{dP}_{u, j}(T \leq t)=\frac{1}{\delta} L_{j}(u, \delta) .
$$

Therefore, we have

$$
P_{u, j}(T \leq t) \simeq \sum_{k=1}^{m} \varpi(k, m) \widetilde{L}_{k, j}(t),
$$

where $\varpi(k, m)$ is given by Eq. (5.5) and

$$
\widetilde{L}_{k, j}(t)=\frac{(2 k) !}{k !(k-1) !} \sum_{i=0}^{k}(-1)^{i}\left(\begin{array}{c}
k \\
i
\end{array}\right) \frac{1}{k+i} L_{j}\left(u,(k+i) \frac{\ln 2}{t}\right), j=1,2 .
$$

In the rest of this section, we assume that $X(0)=e_{1}$.

We arbitrarily choose $\left(\lambda_{11}, \lambda_{12}\right)=(3,6),\left(\lambda_{21}, \lambda_{22}\right)=(5,4), p_{11}=p_{22}=1, \beta_{1}=1.2$ and $\beta_{2}=0.8$. By Corollary 3.1 we can obtain numerical solutions for the distribution of $N_{t}$ and the ruin probabilities $P\left(T_{j} \leq t\right), j=1,2$. From Eqs. (4.21) and (5.8) we obtain numerical results for the ruin probability $P_{u, 1}(T \leq t)$.

In Figure 1, we set $\theta=0.25, a_{11}=1.2, a_{22}=1.2$, and we choose the initial reserves $u_{1}=6.9247$ and $u_{2}=4.9511$ such that $\Psi_{1}\left(u_{1}, 1\right)=P\left(T_{1} \leq 1\right)=0.05$ and $\Psi_{2}\left(u_{2}, 1\right)=$ $P\left(T_{2} \leq 1\right)=0.05$, which give the fair conditions for comparing the numerical values. Figure 1 numerically shows the desired relationships among the distribution of $N_{t}$ and the ruin probabilities $\Psi_{j}\left(u_{j}, t\right)$ 's. For example, as shown in Figure $1, P\left(N_{t}=0\right)>$ $P\left(N_{t}=1\right)>\Psi_{1}\left(u_{1}, t\right)$ (or $\left.>\Psi_{2}\left(u_{2}, t\right)\right)>P\left(N_{t}=2\right)$. This is consistent with our intuition. 


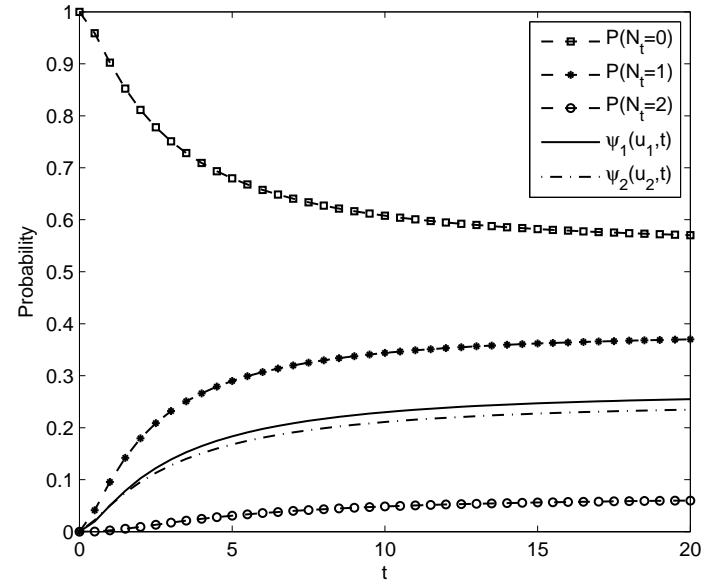

Figure 1: Relationships among the ruin probabilities

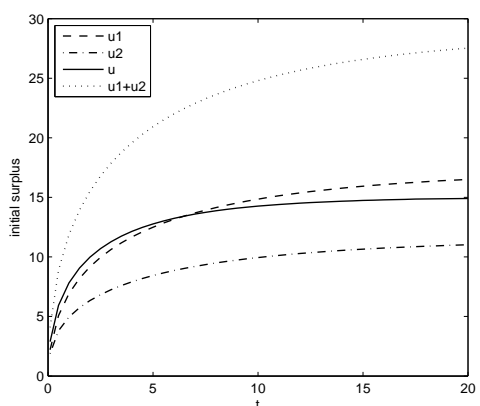

(a) Given $\alpha=0.05$

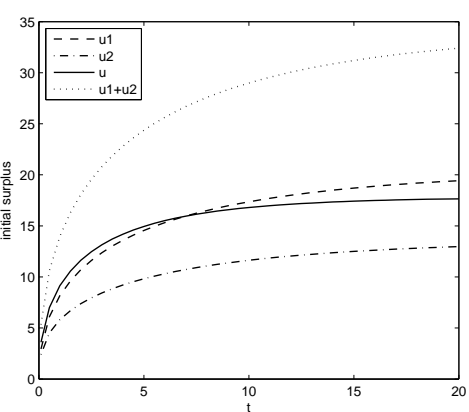

(b) Given $\alpha=0.03$

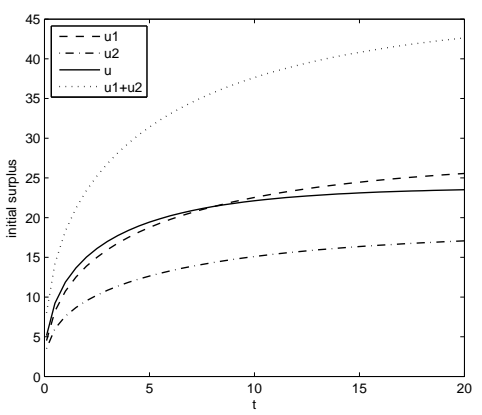

(c) Given $\alpha=0.01$

Figure 2: Differences between initial reserves 
In Fig 2 (a)-(c), we set $\theta=0.25, a_{11}=a_{22}=1.2$. For given solvency level $\Psi_{1}\left(u_{1}, t\right)=$ $\Psi_{2}\left(u_{2}, t\right)=\Psi(u, t)=\alpha$, Figure 2 (a)-(c) numerically present the differences among the initial reserves $u_{1}, u_{2}$ and $u$. From these figures we see that $u>u_{1}$ and $u>u_{2}$ at earlier period. However, with the time lapse, we have $u_{2}<u<u_{1}$. We present these three figures in order to show the differences of the sizes of the initial reserves in scale. For example, the following Table 1 shows the detailed differences of the initial reserves at time $\mathrm{t}=1$.

\begin{tabular}{|c|c|c|c|}
\hline & $\alpha=0.05$ & $\alpha=0.03$ & $\alpha=0.01$ \\
\hline$u_{1}$ & 6.9247 & 8.1650 & 10.7415 \\
\hline$u_{2}$ & 4.9511 & 5.8105 & 7.5847 \\
\hline$u$ & 7.8161 & 9.1417 & 11.8771 \\
\hline
\end{tabular}

Table 1: Initial reserves for given solvency level at $t=1$

In Fig 3 (a)-(c), we set $a_{11}=a_{22}=1.2$. For given solvency level $\Psi_{1}\left(u_{1}, 1\right)=$ $\Psi_{2}\left(u_{2}, 1\right)=\Psi(u, 1)=\alpha$, Figure 3 (a)-(c) numerically present the evolutions of the initial reserves with the increasing of the safety loading coefficient. Since the ruin probability is a decreasing function of the initial reserve and the safety loading coefficient, the initial reserves are decreasing when the safety loading coefficient increases, given the solvency level $\alpha$. Figures 3 (a)-(c) illustrate the differences among the sizes of the initial reserves. The safety loading coefficient $\theta$ is usually determined from the utility of the wealth of the insured and of the insurer. The insurance company could increase the competition of its insurance business by selecting appropriate initial reserve and the safety loading coefficient under given solvency level.

In Fig 4 (a)-(c), we set $\theta=0.25, a_{11}=a_{22}=1.2$. For given solvency level $\alpha$, we choose the initial reserves $u_{1}, u_{2}$ and $u$ as shown in Table 1 , such that $\Psi_{1}\left(u_{1}, 1\right)=$ $\Psi_{2}\left(u_{2}, 1\right)=\Psi(u, 1)=\alpha$. Figure 4 (a)-(c) numerically present the differences among the ruin probabilities. As shown in these figures, we have $\Psi(u, t)>\psi_{1}\left(u_{1}, t\right)>\psi_{2}\left(u_{2}, t\right)>$ $\Psi\left(u_{1}+u_{2}, t\right)$ for $t>1$.

From Figures 1, 2 (a)-(c) and 4 (a)-(c) we see that the combination of two classes of insurance business can reduce the initial reserve under given solvency level. We observe different relationships among ruin probabilities in these figures because we have chosen different initial reserves for same solvency level. The numerical results shown in these figures do not conflict with the conclusions obtained in Section 3.

In Figure 5 , we let $\theta=0.25, a_{11}=a_{22}=1.2$. We choose the same initial reserve by letting $u_{1}=u_{2}=u=5$. Figure 5 shows that $\Psi(u, t)>\Psi_{1}\left(u_{1}, t\right)$ and $\Psi(u, t)>\Psi_{2}\left(u_{2}, t\right)$ in a short period at earlier time and $\Psi_{1}\left(u_{1}, t\right)>\Psi(u, t)>\Psi_{2}\left(u_{2}, t\right)$ for all sufficient large $t$. This does not contradict to Theorem 2.4. Note that the upper bound on the right hand side in inequality (2.15) contain two parts: the exponential function $e^{-r u}$ and the coefficients $H(r)$. We could choose $0<\varepsilon<R$. Even if $e^{-(R-\varepsilon) u}>e^{-R_{j} u}$ for some $R_{j}$, but, we may have $H(R-\varepsilon) e^{-(R-\varepsilon) u} \leq e^{-R_{j} u}$ for some sufficient small $\varepsilon>0$ and initial reserve $u$. 


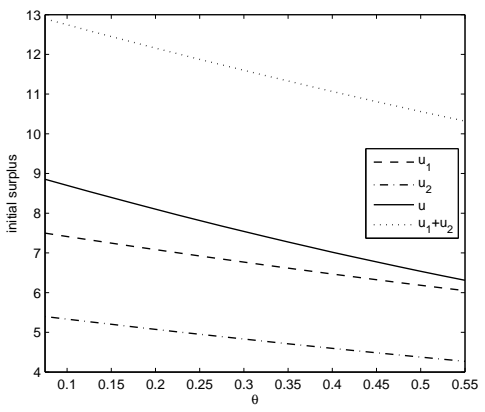

(a) Given $\alpha=0.05$

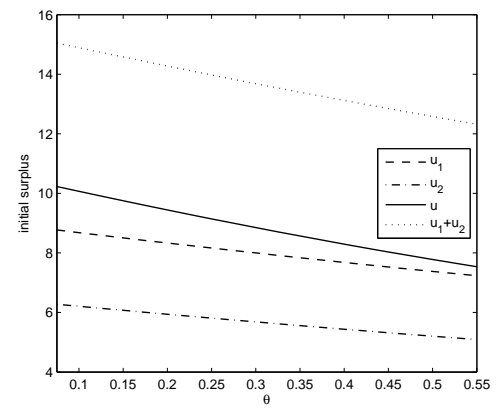

(b) Given $\alpha=0.03$

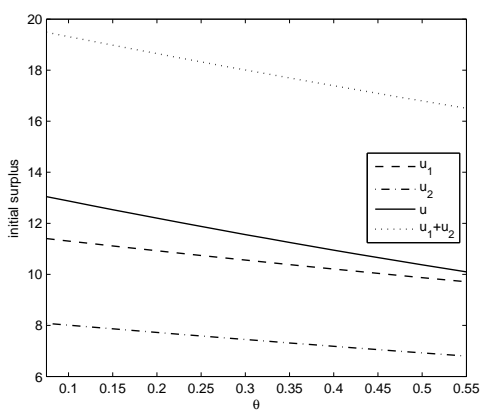

(c) Given $\alpha=0.01$

Figure 3: Relationships among $u_{j}{ }^{\prime}$ s, $u$ and $\theta$

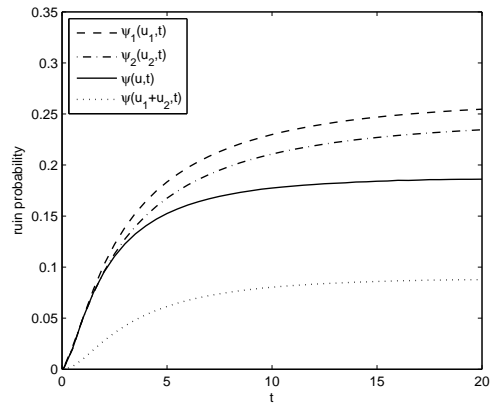

(a) Given $\alpha=0.05$

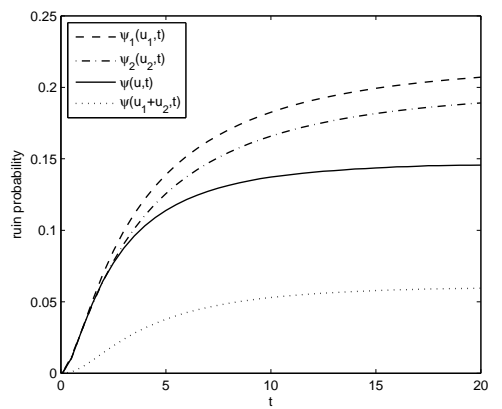

(b) Given $\alpha=0.03$

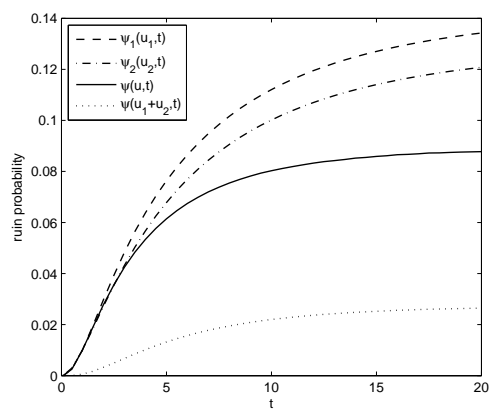

(c) Given $\alpha=0.01$

Figure 4: Relationships among ruin probabilities 


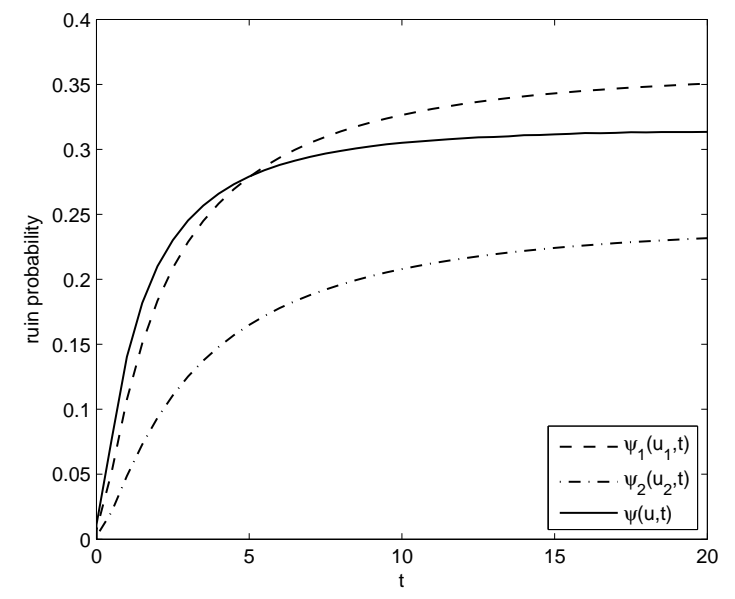

Figure 5: Relationships among ruin probabilities with same initial reserve

Acknowledgement The authors cordially thank the anonymous reviewers and editors for valuable comments to improve the earlier version of the paper. The research of Guojing Wang is supported by the National Natural Science Foundation of China (Grant No. 11371274). The research of Hailiang Yang is supported by the Research Grants Council of the Hong Kong Special Administrative Region (project No. HKU705313P) and a Society of Actuarial CAE Research Grant.

\section{References}

1 Albrecher, H., Boxma, O. J., 2005. On the discounted penalty function in a Markov-dependent risk model. Insurance: Mathematics and Economics 37, 650-672.

2 Albrecher, H., Asmussen, S. 2006. Ruin probabilities and aggregate claims distributions for shot noise Cox processes. Scandinavia Actuarial Journal, 86-110.

3 Ambagaspitiya, R.S., 2003. Aggregate survival probability of a portfolio with dependent subportfolios. Insurance: Mathematics and Economics 32, 431-443.

4 Asmussen, S., Albrecher, H. 2010. Ruin Probabilities. World Scientific Co. Singapore.

5 Chan, W.S, Yang, H., Zhang, L., 2003. Some results on the ruin probability in a two-dimensional risk model. Insurance: Mathematics and Economics 32, 345-358.

6 Dang, L., Zhu, N., Zhang, H., 2009. Survival probability for a two-dimensional risk model. Insurance: Mathematics and Economics 44, 491-496.

7 Dong, Y., Wang, G., Wu, R., 2011. Pricing the zero-coupon bond and its fair premium under a structural credit risk model with jumps. Journal of Applied Probability 48, 404-419.

8 Elliott, R.J., Aggoun, L., Moore, J.B., 1994. Hidden Markov Models: Estimation and Control. Springer-Verlag, New York 
9 Elliott, R.J., Chan, L., Siu, T.K., 2005. Option pricing and Esscher transform under regime switching. Annals of Finance 1 (4), 423-432.

10 Gerber, H.U., 1973. Martingales in risk theory, Mutteilungen der Schweizer Vereinigung der Versicherungs mathematiker, 205-216.

11 Giesecke, K., 2003. A simple exponential model for dependent defaults. Journal of Fixed Income $13(3), 74-83$.

12 Grandell, J., 1991. Aspects of Risk Theory. Springer-Verlag, New York.

13 Kou, S., Wang, H., 2003. First passage times of a jump diffusion process. Advances in Applied Probability 35, 504-531.

14 Kovchegova, Y., Meredith, N., Nir, E. 2010. Occupation times and Bessel densities. Statistics and Probability Letters 80, 104-110

15 Liang, X., Wang, G., 2012. On a reduced form credit risk model with common shock and regime switching. Insurance Math. Econom. 51, 567-575.

16 Lin, X.S., Tan, K., Yang, H., 2009. Pricing annuity guarantees under a regime switching model. North American Actuarial Journal 13 (3), 316-338.

17 Lindskog, F., and McNeil, A., 2003. Common Poisson shock models: applications to insurance and credit risk modelling. Astin Bulletin, 33(2): 209-238.

$18 \mathrm{Lu}, \mathrm{Y} ., \mathrm{Li}, \mathrm{S} ., 2005$. On the probability of ruin in a Markov-modulated risk model. Insurance: Mathematics and Economics 37, 522-532.

$19 \mathrm{Ng}$, A. C. Y., Yang, H., 2006. On the joint distribution of surplus before and after ruin under a Markovian regime switching model. Stochastic Processes and their Applications 116, 244-266.

20 Wang, G., Yuen, K.C., 2005. On a correlated aggregate claims model with thinning-dependence structure. Insurance: Mathematics and Economics 36, 456-468.

21 Zhu, J., Yang, H., 2008. Ruin theory for a Markov regime-switching model under a threshold dividend strategy. Insurance: Mathematics and Economics 42, 311-318. 